\title{
The viral Tat protein has no essential function in HIV-I replication other than to activate transcription
} Atze T Das*, Alex Harwig and Ben Berkhout

\author{
Address: Laboratory of Experimental Virology, Department of Medical Microbiology, Center for Infection and Immunity Amsterdam (CINIMA), \\ Academic Medical Center of the University of Amsterdam, The Netherlands \\ * Corresponding author
}

from Frontiers of Retrovirology: Complex retroviruses, retroelements and their hosts

Montpellier, France. 21-23 September 2009

Published: 24 September 2009

Retrovirology 2009, 6(Suppl 2):P24 doi:10.1186/1742-4690-6-S2-P24

This abstract is available from: http://www.retrovirology.com/content/6/S2/P24

(c) 2009 Das et al; licensee BioMed Central Ltd.

It is generally accepted that the Tat protein has a pivotal role in HIV-1 replication because it activates transcription from the viral LTR promoter through binding of the TAR hairpin in the nascent RNA transcript. However, several other functions of Tat have been suggested in e.g. HIV-1 RNA translation, reverse transcription and RNAi suppression. How important these functions are in viral replication is difficult to determine in replication studies with Tat-mutated HIV-1 variants because of the dominant effect of Tat inactivation on gene expression. We therefore used an HIV-1 variant that does not depend on the Tat/ TAR components for transcription to reassess whether or not Tat has a second essential function in HIV-1 replication. This HIV-rtTA variant uses the incorporated Tet-On gene expression system for activation of transcription. We previously showed that complete deletion of TAR is compatible with HIV-rtTA replication. Complete inactivation of Tat does however inhibit HIV-rtTA replication and further analysis revealed that Tat stimulates transcription via sequence elements in the U3 promoter region. In agreement with this, evolution of the Tat-minus virus in longterm cultures resulted in U3 changes that increased promoter activity. We generated a novel HIV-rtTA variant that lacks HIV-1 U3 promoter and TAR sequences. This virus replicates efficiently without Tat and spontaneously acquired Tat-inactivating mutations in long-term cultures. Our results show that Tat does not only activate HIV-1 transcription through binding to the TAR RNA hairpin but also through an interaction via U3 DNA elements. Moreover, these results demonstrate that Tat has no other essential function during HIV-1 replication in T cell lines in vitro. 